\title{
Why multisystem inflammatory syndrome in children has been less commonly described in Asia?
}

\author{
Weiguo Li ${ }^{1,2,3}$, Yuyi Tang ${ }^{1,2,3}$, Yuan Shi ${ }^{1,2,3}$, Yaolong Chen ${ }^{4,5,6}$, Enmei Liu ${ }^{1,2,3}$ \\ ${ }^{1}$ Department of Respiratory Medicine, Children's Hospital of Chongqing Medical University, Chongqing, China; ${ }^{2}$ National Clinical Research \\ Center for Child Health and Disorders, Ministry of Education Key Laboratory of Child Development and Disorders, China International Science \\ and Technology Cooperation Base of Child Development and Critical Disorders, Children's Hospital of Chongqing Medical University, Chongqing, \\ China; ${ }^{3}$ Chongqing Key Laboratory of Pediatrics, Chongqing, China; ${ }^{4}$ Lanzhou University Institute of Health Data Science, Lanzhou, China; \\ ${ }^{5}$ Evidence-Based Medicine Center, School of Basic Medical Sciences, Lanzhou University, Lanzhou, China; ${ }^{6}$ Key Laboratory of Evidence Based \\ Medicine and Knowledge Translation of Gansu Province, Lanzhou, China \\ Correspondence to: Enmei Liu, MD, PhD. Department of Respiratory Medicine, Children's Hospital of Chongqing Medical University, Chongqing, \\ China. Email: emliu186@126.com.
}

Submitted May 30, 2020. Accepted for publication Oct 16, 2020.

doi: $10.21037 /$ tp-20-151

View this article at: http://dx.doi.org/10.21037/tp-20-151

Recently, pediatricians from Europe and North America have found that increasing cases of multisystem inflammatory syndrome in children (MIS-C) may be associated with SARS-CoV-2 infection (1-3). It is quite confusing that in Asia where Coronavirus Disease 2019 (COVID-19) first outbroke, cases of MIS-C have been less commonly described. Various speculations have then emerged on this issue, and some even suspected that Asian pediatricians missed the diagnosis of MIS-C in the early epidemic, yet we hold different viewpoints on this issue.

In the early epidemic when the published data on COVID-19 in children were limited, most case reports originated from Asia (mainly from China, Figure 1). As early as February 2020, Chinese pediatricians reported the first severe COVID-19 case in children in the world. The patient had prominent gastrointestinal symptoms at onset and rapidly progressed to shock, acute respiratory distress syndrome (ARDS), and kidney failure. We now consider it a suspicious case of MIS-C as the patient had multiple organs involvement (4). Another case series included 171 confirmed cases of children with SARS-CoV-2 infection and only 3 of them required intensive care support and invasive mechanical ventilation (5). All these 3 patients had coexisting conditions and one 10-month-old child died with multiorgan failure. Also, the largest case series on children with COVID-19 was published from China, disclosing detailed symptoms and outcomes of the confirmed cases (6). However, limited number of clustered cases were not enough to conclude the connection with MIS-C. We therefore postulated about the reasons why MIS-C has been less commonly described in Asia.

Firstly, it is probably related to the different prevalence rates of COVID-19. Wuhan, the worst affected city by SARS-CoV-2 in China, has an infection rate of around $0.6 \%$ and a fatality rate of $7.7 \%$, which was much lower than that of the main European epidemic cities, where the infection rate reached up to $9 \%$ (Bergamo, Italy, gathering site of MIS-C cases) and the fatality rate was close to $20 \%$ widely. Children cases may account for less than $2 \%$ of all cases all over the world, and the association might be found only when the prevalence rate was relatively high. Besides, differences in ethnic or genetic background and SARS-CoV-2 subtypes may lead to different COVID-19 prevalence and incidence of MIS-C, but the relationship between ethnicity and susceptibility of MIS-C was inconclusive. Despite cohorts from Europe and North America have showed high proportion of children of black origin, there were certain amounts of Asian patients (2).

During the COVID-19 pandemic, hospitals in China conducted large-scale SARS-CoV-2 screening for patients with fever, especially for those with severe illness. Other Asian countries were likely to have adopted the same strategy, which would be an essential opportunity for catching COVID-19 related syndromes. However, no clustered MIS-C cases like those in the Italian pediatric intensive care unit (PICU) were observed. MIS-C presents 


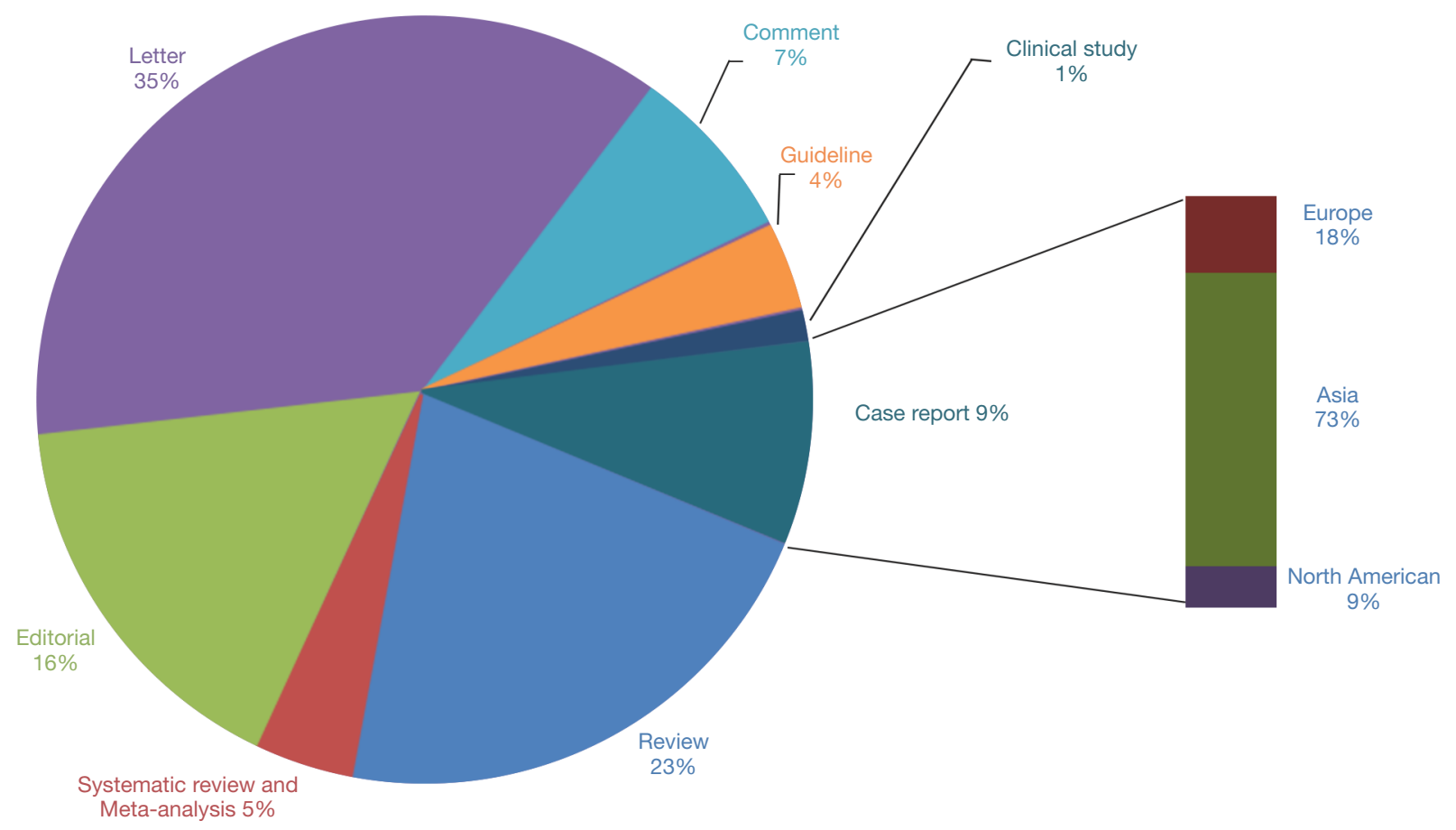

Figure 1 Types of published articles on children with Coronavirus Disease 2019 (COVID-19) (n=249). Data based on our PubMed search from its inception to May 22, 2020 (The details of the search strategy can be found in Appendix 1). It indicated that at the onset of the outbreak, most case reports were from Asia.

with symptoms similar to Kawasaki disease (KD), which is first detected and named in Japan (7). Moreover, as the country with the highest incidence of reported KD in children, Japan is a candidate country to identify the association between SARS-CoV-2 infection and MIS-C. A possible association between the coronavirus infection and KD was first reported as long ago as 2005 in New Haven, USA (8). In order to prove the hypothesis again, Japanese researchers performed multiple studies but unfortunately failed to confirm it. In addition, Tokyo, the worst affected city in Japan, has an infection rate of only $0.1 \%$ and a case fatality rate of $4.4 \%$. As a reportable disease in Japan, $\mathrm{KD}$ or related syndromes are surveilled by their health care system, and these data will add more evidence for the association.

The possible association between MIS-C and SARS$\mathrm{CoV}-2$ infection is a rather potential risk for children. As advocated by WHO, pediatricians around the world should strengthen the surveillance of MIS-C during the COVID-19 pandemic (9). Serological and PCR tests for SARS-CoV-2 should be carried out in suspected MIS-C cases, and those children should also be recruited in regional or international clinical researches, like the DIAMONDS study funded by Europe Union. At present, we are facing a challenging problem, that is, understanding how SARS$\mathrm{CoV}-2$ infection affects the immune system, which is critical for the development of vaccines and drugs, as well as the prediction of the risk of vaccine side effects. It's worth mentioning that recently we have conducted a guideline for COVID-19 in children, which recommends against using intravenous immunoglobulin (IVIG) to treat children with severe COVID-19 (10). We may now need to re-evaluate the evidence and update our recommendation on IVIG, taking into consideration that more and more children are being affected by MIS-C.

\section{Acknowledgments}

Funding: National Clinical Research Center for Child Health and Disorders (Children's Hospital of Chongqing Medical University, Chongqing, China) (NCRCCHD2020-EP-01); The fourth batch of "Special Project of Science and Technology for Emergency Response to COVID-19" of Chongqing Science and Technology Bureau; Special Fund for Key Research and Development Projects in Gansu Province in 2020. 


\section{Footnote}

Peer Review File: Available at http://dx.doi.org/10.21037/tp20-151

Conflicts of Interest: All authors have completed the ICMJE uniform disclosure form (available at http://dx.doi. org/10.21037/tp-20-151). The authors have no conflicts of interest to declare.

Ethical Statement: The authors are accountable for all aspects of the work in ensuring that questions related to the accuracy or integrity of any part of the work are appropriately investigated and resolved.

Open Access Statement: This is an Open Access article distributed in accordance with the Creative Commons Attribution-NonCommercial-NoDerivs 4.0 International License (CC BY-NC-ND 4.0), which permits the noncommercial replication and distribution of the article with the strict proviso that no changes or edits are made and the original work is properly cited (including links to both the formal publication through the relevant DOI and the license). See: https://creativecommons.org/licenses/by-nc-nd/4.0/.

\section{References}

1. Verdoni L, Mazza A, Gervasoni A, et al. An outbreak of severe Kawasaki-like disease at the Italian epicentre of the SARS-CoV-2 epidemic: an observational cohort study. Lancet 2020;395:1771-8.

Cite this article as: Li W, Tang Y, Shi Y, Chen Y, Liu E. Why multisystem inflammatory syndrome in children has been less commonly described in Asia? Transl Pediatr 2020;9(6):873-875. doi: $10.21037 /$ tp-20-151
2. Whittaker E, Bamford A, Kenny J, et al. Clinical Characteristics of 58 Children With a Pediatric Inflammatory Multisystem Syndrome Temporally Associated With SARS-CoV-2. JAMA 2020;324:259-69.

3. Swann OV, Holden KA, Turtle L, et al. Clinical characteristics of children and young people admitted to hospital with covid-19 in United Kingdom: prospective multicentre observational cohort study. BMJ 2020;370:m3249.

4. Chen F, Liu ZS, Zhang FR, et al. [First case of severe childhood novel coronavirus pneumonia in China. Zhonghua Er Ke Za Zhi 2020;58:E005.

5. Lu X, Zhang L, Du H, et al. SARS-CoV-2 Infection in Children. N Engl J Med 2020;382:1663-5.

6. Dong Y, Mo X, Hu Y, et al. Epidemiology of COVID-19 Among Children in China. Pediatrics 2020;145:e20200702.

7. Kawasaki T. [Acute febrile mucocutaneous syndrome with lymphoid involvement with specific desquamation of the fingers and toes in children. Arerugi 1967;16:178-222.

8. Esper F, Shapiro ED, Weibel C, et al. Association between a novel human coronavirus and Kawasaki disease. J Infect Dis 2005; 191:499-502.

9. Multisystem inflammatory syndrome in children and adolescents with COVID-19. Scientific brief. 15 May 2020. Available online: https://www.who.int/newsroom/commentaries/detail/multisystem-inflammatorysyndrome-in-children-and-adolescents-with-covid-19

10. Liu E, Smyth RL, Luo Z, et al. Rapid advice guidelines for management of children with COVID-19. Ann Transl Med 2020;8:617. 


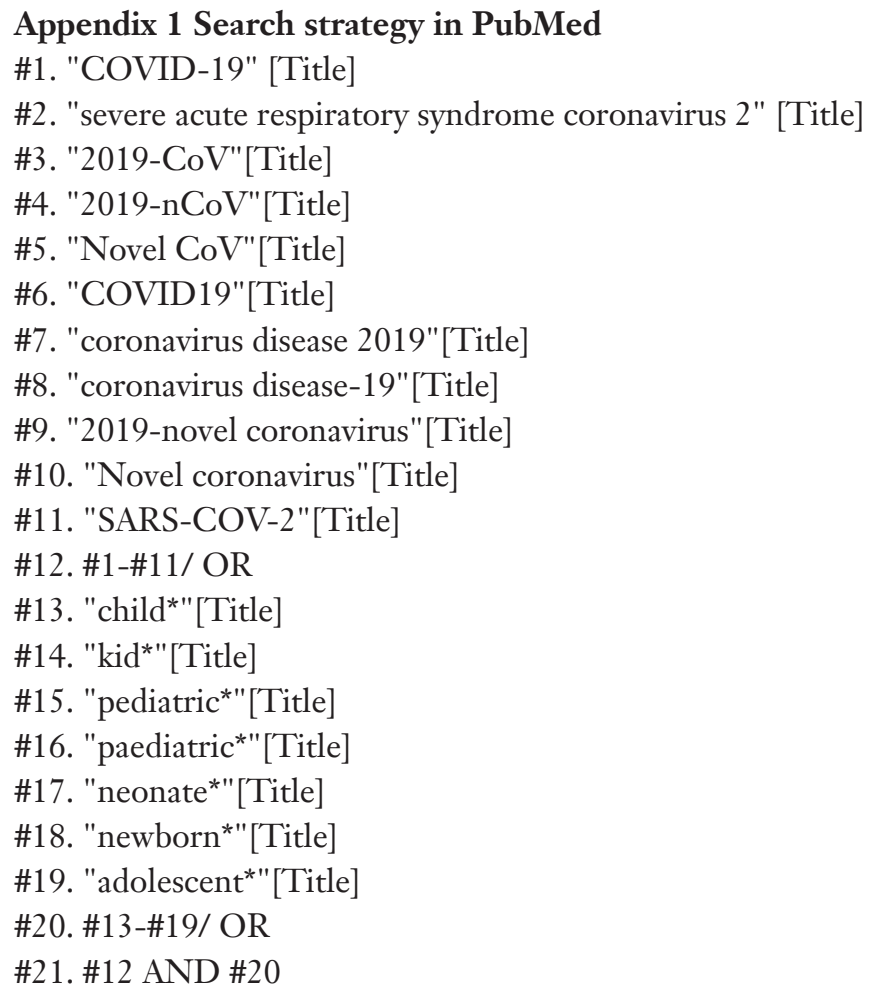

